\title{
Retrospective study of the efficacy and toxicity of lobaplatin in combined chemotherapy for metastatic breast cancer
}

This article was published in the following Dove Press journal: OncoTargets and Therapy

\author{
Yuan $\mathrm{Wu}^{*}$ \\ Xiao-Yue Xu* \\ Fei Yan \\ Wei-Li Sun \\ Yan Zhang \\ De-Lin Liu* \\ Bo Shen*
}

Department of Oncology, Jiangsu Cancer Hospital \& Jiangsu Institute of Cancer Research \& Nanjing Medical University Affiliated Cancer Hospital, Nanjing, People's Republic of China

*These authors contributed equally to this work
Correspondence: De-Lin Liu; Bo Shen Jiangsu Cancer Hospital \& Jiangsu Institute of Cancer Research \& Nanjing Medical University Affiliated Cancer Hospital, Baiziting No. 42, Nanjing, Jiangsu Province 210009, People's Republic of China Fax +8602583283554

Email delinn@sohu.com; 13913910555@126.com
Objective: To study the efficacy and adverse reactions of lobaplatin combined with other chemotherapy drugs in the treatment of metastatic breast cancer.

Methods: This retrospective analysis enrolled 114 patients who were diagnosed with advanced breast cancer from January 2010 to December 2015. Lobaplatin and another chemotherapeutic agent were given to patients. The efficacy and side effects were evaluated after at least two cycles of chemotherapy.

Results: Therapeutic efficacy and adverse reactions could be evaluated in 112 patients with 2 complete response (CR) patients, 31 cases of partial response (PR), 52 cases of stable disease (SD) and 27 cases of progressive disease (PD). The overall response rate (ORR) was $29.5 \%$ and the disease control rate (DCR) was $75.9 \%$. The median time to progression (TTP) was 7.7 months, and the median overall survival (OS) was expected to be 28.0 months. The main side effects were myelosuppression. Twenty five patients $(21.9 \%)$ had grade $3 / 4$ neutrophil suppression, 18 patients $(15.8 \%)$ had grade $3 / 4$ thrombocytopenia. Other toxicities included gastrointestinal reaction, peripheral neuropathy, stomatitis, hepatic dysfunction, fatigue and skin rashes, which were alleviated by symptomatic treatment.

Conclusion: Lobaplatin-based regimen chemotherapy for advanced metastatic breast cancer patients is effective and well tolerated.

Keywords: metastatic breast cancer, lobaplatin, chemotherapy, side effects, retrospective study

\section{Introduction}

According to research reports from International agency for research on cancer, breast cancer is still ranking first with regard to the occurrence among female malignant tumors. During the past three decades, the occurrence rate of breast cancer in many countries showed a progressive increasing trend year-by-year. Even new therapies including targeted therapy, endocrine therapy, immunotherapy and others have become focus and trends of recent studies. However, chemotherapy remains the base of salvage treatment for advanced metastatic breast cancer (MBC) especially in patients with drug resistance for endocrine and targeted therapies, rapid progression, visceral crisis and triple negative breast cancer. With the widespread use of a combination of anthracyclines and paclitaxel (also including docetaxel and nab-paclitaxel), the treatment of breast cancer has made great progress, and it has also led to an increase in the proportion of resistance to these two types of drugs. There is no accepted alternative therapy for patients who have had 
recurrence and metastasis after treatment with anthracyclines and taxanes. ${ }^{1}$ Medication options cover capecitabine, gemcitabine, docetaxel, nab-paclitaxel, vinorelbine, platinum-based medications and others for single-agent or combined chemotherapy. It was indicated in initial studies that platinum anticancer drugs, as single-agent or combined treatment, could achieve favorable therapeutic effect in $\mathrm{MBC} .^{2-7}$ However, the application of platinum-based medication was restricted for severe renal toxicity, ototoxicity, neurotoxicity and gastrointestinal adverse reaction as well as potential acute allergic reaction and significant myelosuppression. ${ }^{8,9}$ Lobaplatin is the third-generation platinum-based anticancer medication. It was indicated that favorable outcomes were achieved with application of lobaplatin in MBC. ${ }^{10-12}$ A total of 114 recurrent $\mathrm{MBC}$ cases who received lobaplatin-combined therapy in Jiangsu Cancer Hospital and Research Institute from January 2010 to December 2014 were analyzed retrospectively with observations of therapeutic effects and adverse reactions. The report results are as follows.

\section{Materials and methods}

\section{General information}

Women with histological or cytological confirmed bidimensionally measurable breast cancer with clinical evidence of metastatic disease were eligible for this study. A total of 114 invasive breast cancer patients (range 34-74 years; median age of 52) who were recruited from Jiangsu Tumor Hospital and Research Institute between January 2010 and December 2014 were enrolled. Most cases were involved with multiple organs. There are 73 cases with lymphatic metastasis, 48 with pulmonary metastasis, 52 with liver metastasis, 62 with bone metastasis, 18 with brain metastasis and 11 with skin and softtissue metastasis. A total of 41 patients received first-line treatment, among which 2 received chemotherapy for one cycle without evaluation of therapeutic outcome, 29 received second-line treatment and 44 received third-line therapy and the above. The ECOG scores of the patients ranged from 0 to 1 (Table 1 ).

\section{Methods}

Lobaplatin (provided by Yibai Pharmaceutical Company) Application: $30 \mathrm{mg} / \mathrm{m}^{2} \mathrm{~d} 2$; the medications for lobaplatin-combined therapy include pemetrexed (PEM) of 500 $\mathrm{mg} / \mathrm{m}^{2} \mathrm{~d} 1 \quad(\mathrm{n}=23)$, Navelbine $(\mathrm{NVB})$ of $25 \mathrm{mg} / \mathrm{m}^{2} \mathrm{~d} 1 \&$ $\mathrm{d} 8(\mathrm{n}=18)$, Docetaxel (DOC) of $75 \mathrm{mg} / \mathrm{m}^{2} \mathrm{~d} 1 \quad(\mathrm{n}=15)$,
Taxol (TAX) of $175 \mathrm{mg} / \mathrm{m}^{2} \mathrm{~d} 1(\mathrm{n}=41)$ and Gemcitabine (GEM) of $1,000 \mathrm{mg} / \mathrm{m}^{2} \mathrm{~d} 1 \& \mathrm{~d} 8(\mathrm{n}=17)$. Every cycle takes 3 weeks. Repeated imaging was performed every 2 cycles for evaluation of therapeutic effect. Patients presented with therapeutic achievements $(\mathrm{CR}+\mathrm{PR}+\mathrm{SD})$ continued original chemotherapy until disease progression, unacceptable toxicity. Ones with progressive conditions received alternative therapies. Before chemotherapy, conventional application of 5-HT3 receptor antagonist was adopted for preventive antiemetic therapy.

\section{Clinical workup}

The evaluation of therapeutic effect was based on the RECIST 1.1 (2009). Complete remission (CR) refers to conditions with all target foci disappeared; partial remission (PR) refers to conditions with a total length reduction of $\geq 30 \%$ for baseline foci; progression of disease (PD) refers to conditions with a total length increase of $\geq 20 \%$ for baseline foci or with new foci(s); stable disease (SD) refers to conditions with a total length reduction of below PR level or with a total length increase of below PD level for baseline foci. Imaging (spiral CT) before treatment was performed. Enrolled cases had one or the above measurable foci(s). Repeated imaging and evaluation of therapeutic effect were performed every 2 cycles. If the evaluation result was $\mathrm{CR}$, $\mathrm{PR}$ or $\mathrm{SD}$, the chemotherapy continued. Alternative therapies were performed in patients with PD. Toxicity was evaluated according to the revised CTCAE 4.0 as Grade $0 \sim$ IV.

\section{Statistical analysis}

SPSS 17.0 statistical software was adopted for statistical analysis. Pearson's chi-square test (or Fisher's Exact Test) was adopted for the analysis of enumeration data from different groups. $P<0.05$ indicates statistically significant different. The time to -progression (TTP) and overall survival (OS) were counted in month. Kaplan-Meier method was adopted for survival analysis.

\section{Results \\ Clinical activity}

Overall, 2 patients received treatment of one cycle without therapeutic effect evaluation. A total of 112 patients received treatment of 2 and the above cycles. For these cases, average chemotherapy cycle was 3.98 , objective effective rate $(\mathrm{CR}+\mathrm{PR})$ of the group was $29.5 \%$, while the disease control rate (DCR) $(\mathrm{CR}+\mathrm{PR}+\mathrm{SD})$ was 
Table I Baseline patient characteristics $(n=|| 4)$

\begin{tabular}{|c|c|}
\hline Median age (range) & $52(34-74)$ \\
\hline \multicolumn{2}{|l|}{ ECOG performance status, $\mathrm{n}(\%)$} \\
\hline 0 & $46(40.4)$ \\
\hline I & $68(59.6)$ \\
\hline \multicolumn{2}{|l|}{ Hormonal receptor status, n (\%) } \\
\hline Both ER and PgR negative & $35(30.7)$ \\
\hline ER and (or) PgR positive & $78(68.4)$ \\
\hline ER and PgR status unknown & I (0.9) \\
\hline \multicolumn{2}{|l|}{ HER2 status, n (\%) } \\
\hline HER2 positive & $35(30.7)$ \\
\hline HER2 negative & $78(68.4)$ \\
\hline HER2 unkown & I (0.9) \\
\hline \multicolumn{2}{|l|}{ Metastatic sites, n (\%) } \\
\hline Brain & $18(15.8)$ \\
\hline Lung & $48(42.1)$ \\
\hline Liver & $52(45.6)$ \\
\hline Bone & $62(54.4)$ \\
\hline Lymph nodes & $73(64.0)$ \\
\hline Chest wall or skin & II (9.6) \\
\hline Visceral disease, n (\%) & $89(78.1)$ \\
\hline \multicolumn{2}{|l|}{ Chemotherapy line, $n(\%)$} \\
\hline I & $4 I(36.0)$ \\
\hline 2 & $29(25.4)$ \\
\hline$\geq 3$ & $44(38.6)$ \\
\hline \multicolumn{2}{|l|}{ Drug combination, n (\%) } \\
\hline Gemcitabine & $17(14.9)$ \\
\hline Taxol & $4 \mid(36.0)$ \\
\hline Docetaxel & II (9.6) \\
\hline Vinorelbine & $15(13.2)$ \\
\hline Pemetrexed & $23(20.1)$ \\
\hline Albumin-bound paclitaxel & $6(5.3)$ \\
\hline Liposomal doxorubicin & I (0.9) \\
\hline
\end{tabular}

Abbreviations: PR, partial response; PgR, progesterone receptor.

$75.9 \%$; among which, there were 2 cases (1.8\%) with CR, $31(27.7 \%)$ with PR, 52 (46.4\%) with SD and 27 (24.1\%) with PD. The patients were followed up until December 2017. Median follow-up time was 19 months. During the follow-up period, there were 94 cases $(83.9 \%)$ with disease progression, 62 deaths (55.4\%), 8 lost patients of follow-up and 10 cases without meeting the TTP. Median TTP was 7.7 months (95\% CI: 5.17-10.22). The survival curve was immature. Estimated median OS was 28.0 months (Figures 1-2). Stratification analysis: There were 111 patients with known hormone receptor (HR) condition including 78 HR-positive patients (70.3\%; 1 CR case, 20 PR cases, 41 SD cases; overall response rate (ORR): $26.9 \%$, DCR 79.5\%) and 22 HR- negative patients $(29.7 \% ; 1 \mathrm{CR}$ case, $11 \mathrm{PR}$ cases, $11 \mathrm{SD}$ cases; ORR: $36.4 \%$, DCR 69.7\%). There was not statistically significant difference between the two groups $(P>0.05)$. There were 111 patients with known Her2 status, including 35 Her2-positive cases $(31.5 \%$; 1 CR case, 15 PR cases, 15 SD cases; ORR: 45.7\%, DCR 88.6\%) and 76 Her2-negative cases ( $1 \mathrm{CR}$ case, 16 PR cases, $37 \mathrm{SD}$ cases; ORR: 22.4\%, DCR 71.1\%). Inter-group ORR and DCR showed statistically significant differences $(P=0.012$; $P=0.043)$. For the analysis between the triple negative breast cancer patients (TNBC) group $(n=18)$ or non-triple negative breast cancer patients (Non-TNBC) group $(n=93)$, the ORR and DCR of the TNBC group were $11.1 \%(2 / 18)$ and $50 \%(9 / 18)$, respectively, while the ORR and DCR of the Non-TNBC group were $33.3 \%$ (31/93) and $81.7 \%$ (76/93), respectively. There was a large difference in values between the two groups, but there was no statistically significant difference between the groups (Table 2). The TTP of the two groups was 3.7 months and 9.0 months, respectively, and the difference was significant ( $P=0.003$, Figure 3$)$; but there was no difference between the two groups of OS (30.8 M vs 21.2 M, Figure 4). According to the stratified analysis of different chemotherapy lines, there were 112 cases available for evaluation including 39 cases with first-line therapy (2 CR cases, 13 PR cases, 17 SD cases; ORR: 38.5\%, DCR 82.1\%), 29 cases with second-line therapy (0 CR case, 8 PR cases, 17 SD cases; ORR: $27.6 \%$, DCR $86.2 \%$ ) and 44 cases with third and the above-line therapies (0 CR case, 10 PR cases, 18 SD cases; ORR: 22.7\%, DCR $63.6 \%$ ). No statistically significant difference of ORR and DCR was founding in these three groups; but the TTP survival analysis showed statistical difference obviously (Table 3, Figures 5, 6).

\section{Toxicity}

The common adverse reaction during treatment in the 114 $\mathrm{MBC}$ cases was myelosuppression with major manifestations including thrombocytopenia and neutropenia. Most cases were Grade 1/2 thrombocytopenia and neutropenia. There were 25 cases $(21.9 \%)$ with Grade 3/4 neutropenia and 18 cases $(15.8 \%)$ with Grade $3 / 4$ thrombocytopenia. Among all the patients we enrolled, two patients had neutropenic fever during the course of treatment. However, this symptom was improved after the antipyretic symptomatic treatment. Five patients $(4.5 \%)$ required platelet transfusion for thrombocytopenia. There was no toxicity-related death during treatment; other adverse reaction 


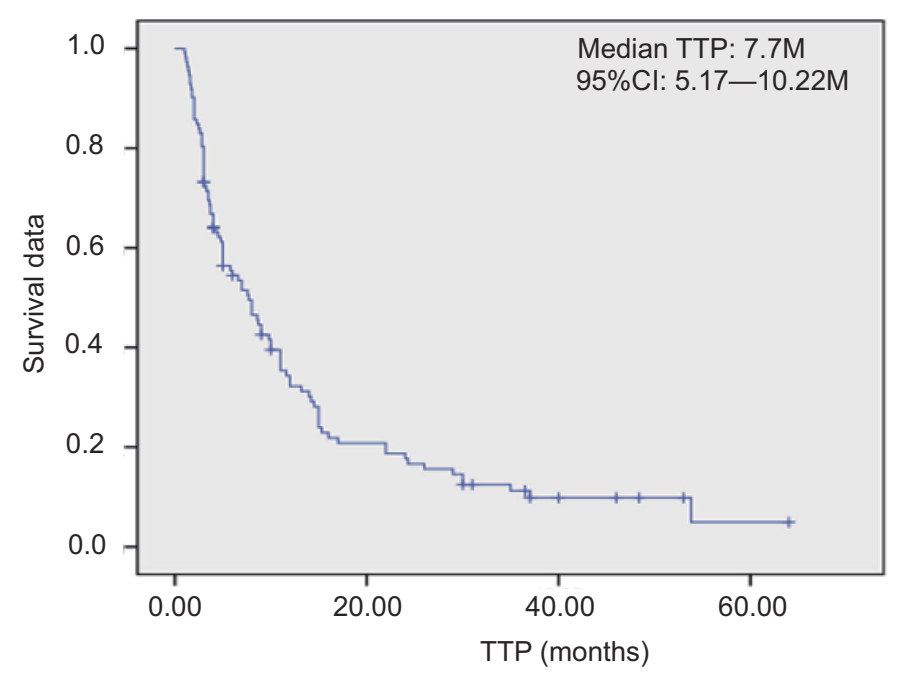

$\rightarrow$ Survival

+ Censor

Figure I Kaplan-Meier plot of Time-to-Progression.

Abbreviation: TTP, time to progression.

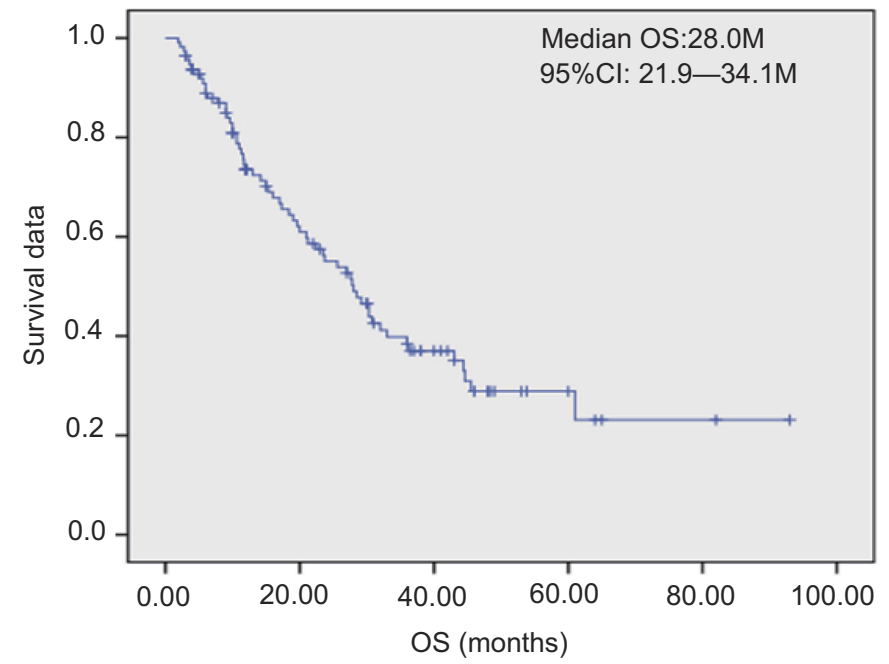

Figure 2 Kaplan-Meier plot of Overall Survival.

Table 2 Comparison of different groups therapeutic effect

\begin{tabular}{|l|l|l|l|l|}
\hline Group & N (\%) & ORR (\%) & DCR (\%) & P \\
\hline HR+ & $78(70.3)$ & $21(26.9)$ & $62(79.5)$ & $>0.05$ \\
HR- & $33(29.7)$ & $12(36.4)$ & $23(69.7)$ & \\
Her2+ & $35(31.5)$ & $16(45.7)$ & $31(88.6)$ & $=0.012$ \\
Her2- & $76(68.5)$ & $17(22.4)$ & $54(71.1)$ & \\
TNBC & $18(16.2)$ & $2(11.1)$ & $9(50.0)$ & $>0.05$ \\
Non-TNBC & $93(83.8)$ & $31(33.3)$ & $76(81.7)$ & \\
\hline
\end{tabular}

Abbreviations: $\mathrm{DCR}$, disease control rate; ORR, overall response rate; TNBC, triple negative breast cancer.

covered fatigue (16.7\%), rash (5.3\%), nausea and vomit $(52.6 \%)$, stomatitis, peripheral neuropathy, mild hepatic dysfunction and others (Table 4).

\section{Discussion}

With the standardization of treatment, the therapeutic effect of breast cancer has been significantly improved. ${ }^{13,14}$ Systematic medication therapies including endocrine therapy, chemotherapy, targeted therapy and others are major therapies for $\mathrm{MBC}$. For $\mathrm{MBC}$ with rapid disease progression, viscera metastasis, skin involvement companied by lymphatic metastasis, below 2 years of disease-free survival, negative or unknown receptor and previous drug resistance for endocrine therapy, chemotherapy reminds the preferred therapy. Especially for visceral crisis patients with rapid disease progression as well as negative hormone receptor or resistance to endocrine therapy, systemic chemotherapy is usually 

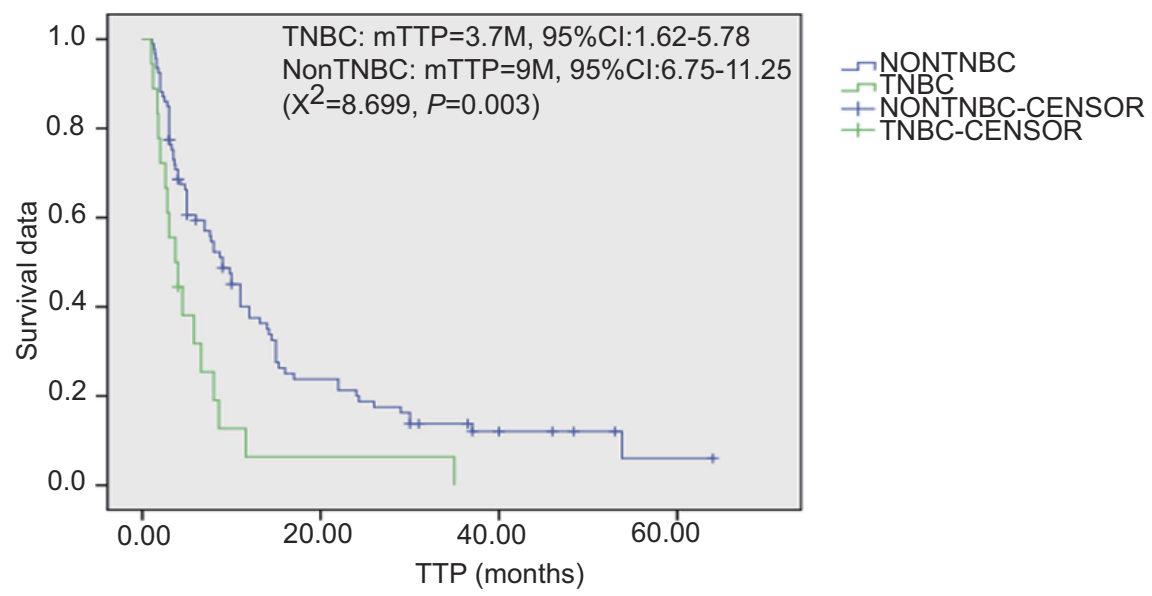

Figure 3 Kaplan-Meier plot of Time-to-Progression for TNBC and non-TNBC.

Abbreviations: TTP, time to progression; TNBC, triple negative breast cancer.
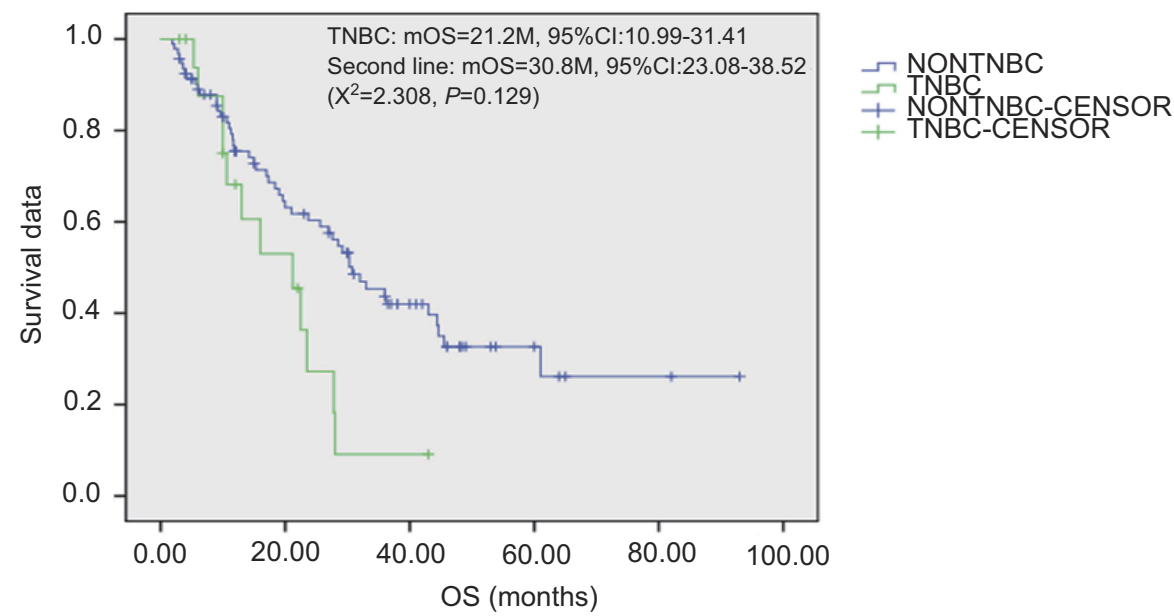

Figure 4 Kaplan-Meier plot of Overall Survival for TNBC and non-TNBC.

Abbreviation: TNBC, triple negative breast cancer.

Table 3 Different line chemotherapy outcomes

\begin{tabular}{|l|l|l|l|l|}
\hline $\begin{array}{l}\text { Chemotherapy } \\
\text { line }\end{array}$ & N (\%) & $\begin{array}{l}\text { ORR } \\
\text { (\%) }\end{array}$ & $\begin{array}{l}\text { DCR } \\
\text { (\%) }\end{array}$ & P \\
\hline First-line & $39(34.8)$ & $15(38.5)$ & $32(82.1)$ & $>0.05$ \\
Second-line & $29(25.9)$ & $8(27.6)$ & $25(86.2)$ & \\
Third or more line & $44(39.3)$ & $10(22.7)$ & $28(63.6)$ & \\
\hline
\end{tabular}

Abbreviations: $\mathrm{DCR}$, disease control rate; ORR, overall response rate.

administered to control the condition, relieve symptoms, improve quality of life and extend survival time. ${ }^{15}$ Anthracene nucleus-contained chemotherapy regimens have become the standard regimen of preoperative neoadjuvant chemotherapy for breast cancer, postoperative adjuvant chemotherapy and MBC treatment. But for late-stage patients with resistance to anthracene nucleus, there is no standard chemotherapy regimen alternatively. According to the guidelines of NCCN, ESMO and Chinese Anti-Cancer Association, optional medications for MBC chemotherapy cover taxanes, navelbine, gemcitabine, capecitabine, platinum-based medications and others. How to achieve reasonable combined application of medications to have maximal therapeutic and reduce adverse reactions to the most requires further study and investigation. The application of lobaplatin-combined therapy in our hospital has achieved favorable outcomes for recent years.

Lobaplatin (chemical name: 1,2-cyclobutanedimethanamine-N,N') is the third-generation platinum-based anticancer compound developed by ASTA Medical (Degussa, German). The anti-tumor mechanism of lobaplatin is similar to other platinum agents, forming DNA intra-strand and/or inter-strand cross-linking and, therefore, affecting the normal 

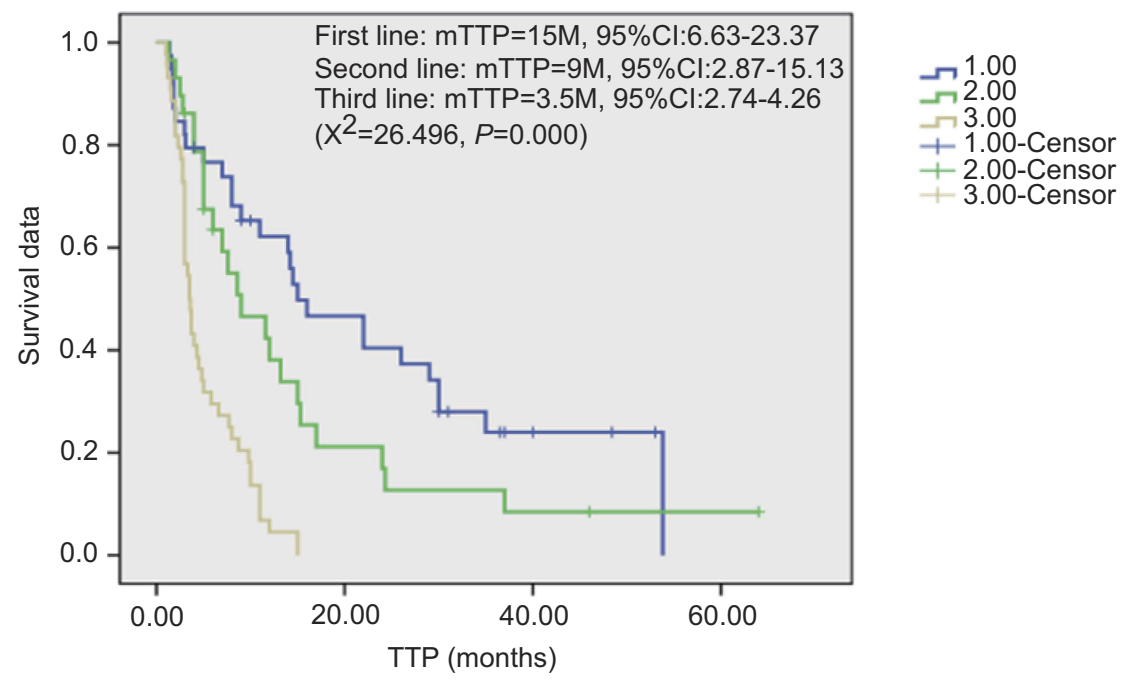

Figure 5 Kaplan-Meier plot of Time-to-Progression for different line therapy. Abbreviation: TTP, time to progression.
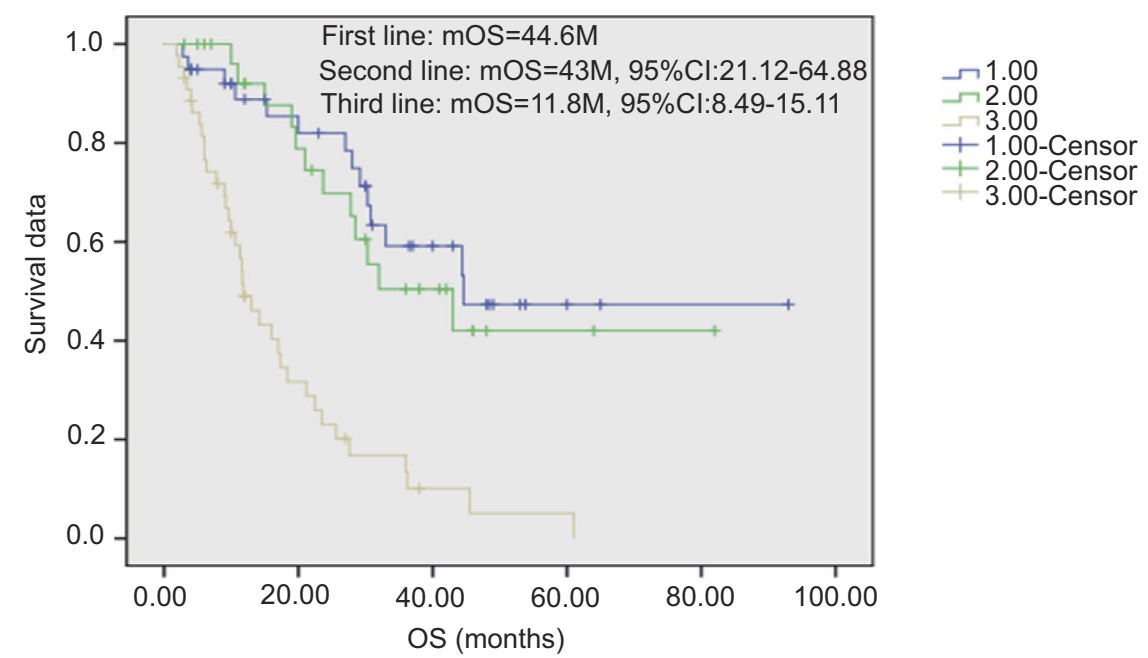

Figure 6 Kaplan-Meier plot of Time-to-Progression for different line therapy.

Table 4 Toxicity of lobaplatin-based regimen in treatment of metastatic breast cancer patients $\mathrm{n}(\%)$

\begin{tabular}{|l|l|l|l|}
\hline Toxicities & Grades I-2 & Grade 3 & Grade 4 \\
\hline Neutropenia & $59(51.8)$ & $18(15.8)$ & $7(6.1)$ \\
Thrombocytopenia & $36(19.2)$ & $12(10.5)$ & $6(5.3)$ \\
Anemia & $22(19.3)$ & $5(4.4)$ & $0(0.0)$ \\
Nausea/Vomiting & $53(46.5)$ & $7(6.1)$ & $0(0.0)$ \\
Diarrhea & $5(4.4)$ & $0(0.0)$ & $0(0.0)$ \\
Rash & $6(5.3)$ & $0(0.0)$ & $0(0.0)$ \\
Stomatitis & $8(7.0)$ & $0(0.0)$ & $0(0.0)$ \\
Peripheral neuropathy & $11(9.6)$ & $0(0.0)$ & $0(0.0)$ \\
Fatigue & $18(15.8)$ & $1(0.9)$ & $0(0.0)$ \\
Anaphylaxis & $3(2.6)$ & $0(0.0)$ & $0(0.0)$ \\
Infection & $3(2.6)$ & $0(0.0)$ & $0(0.0)$ \\
Hepatic dysfunction & $15(13.2)$ & $1(0.9)$ & $0(0.0)$ \\
\hline
\end{tabular}

functions of DNA and achieving the purpose of inhibiting tumor. ${ }^{16}$ It was showed in preclinical studies that the anticancer therapeutic index of lobaplatin equaled or was higher than cisplatin and carboplatin; also, lobaplatin was effective for partial tumors with cisplatin and carboplatin resistance, had lower renal toxicity than cisplatin and incomplete cross resistance with other platinum agents, and showed better anti-tumor activity in preclinical studies. ${ }^{17-19}$ Clinical studies $^{20-27}$ indicated that lobaplatin single-agent therapy, with low toxicity, held certain therapeutic effect in breast cancer, ovarian cancer, head and neck squamous cell carcinoma, lung cancer, urinary transitional epithelium tumor, esophagus cancer, chronic myeloid leukemia and others. However, Neutropenia, anemia, nausea and vomiting are 
observed as side effects. In addition, thrombocytopenia is the most commonly observed dose-limiting toxicity associated with this drug. Therefore, lobaplatin was a broad-spectrum and effective anti-tumor drug with low toxicity. ${ }^{24,28}$ The effective rate of lobaplatin single-agent chemotherapy in advanced breast cancer cases was $45.2 \%$ (the rates for patients without and with previous chemotherapy were $53.5 \%$ and $28.5 \%$, respectively), while the major toxicities were myelosuppression and gastrointestinal reaction. ${ }^{11}$ A China multicenter clinical study for navelbine and lobaplatin combination therapy in advanced breast cancer cases reported a RR of $42.4 \%$ and a DCR of $75.8 \%$. But the occurrence of Grade 3/4 granulocytopenia was as high as $57.6 \%$ and the rate of Grade 3 thrombocytopenia was $9.1 \%{ }^{29}$ Deng Qian-Qian ${ }^{30}$ studied some cases of MBC who were resistant to the combination of pemetrexed and lobaplatin and it was reported that the RR was $15.8 \%$, the DCR was $42.1 \%$ and the median OS was 10.3 months in these patients. After symptomatic treatment, major adverse reactions (myelosuppression and gastrointestinal reactions) were all remitted. A report studied by Wang $\mathrm{ZP}^{31}$ showed that the lobaplatin-based combination regimen is superior to the cisplatin-based combination regimen in the treatment of $\mathrm{MBC}$ after paclitaxel and anthracycline treatment (mPFS 13.2 vs 4.7 months, hazard ratio $=0.37$ ). A Phase II study ${ }^{32}$ showed that the chemotherapy regimen including docetaxel plus epirubicin plus lobaplatin was compared with the docetaxel plus epirubicin regimen for neoadjuvant chemotherapy for breast cancer. The $\mathrm{pCR}$ rate $(38.7 \%$ vs $12.7 \%, P=0.001)$ and ORR (93.5\% vs $73.0 \%, P=0.003)$, and the toxicity of the three drugs combination group also increased significantly. Favorable therapeutic effect and tolerable toxic adverse reactions were reported in various China and foreign studies which reported lobaplatin and docetaxel, vinorelbine, pemetrexed as well as gemcitabine-combined treatment in recurrent $\mathrm{MBC}$ cases. $^{33-36}$

Patients enrolled in this study are all MBC treated with first-line, second-line and third and the above-line therapies. The results were promising with a total effective rate of $29.5 \%$ and a DCR of $75.9 \%$. While adverse reactions were tolerable, major adverse reactions were myelosuppression with $21.9 \%$ grade $3 / 4$ granulocyte reduction and $15.8 \%$ grade 3/4 thrombocytopenia. The occurrence of grade 3/4 myelosuppression was relatively high which might be related with the large amount of patients who received repeated therapies (the rate of patients who received second-line, third-line and the above-line therapy reached 64.0\%) and the relatively large amount of patients $(54.4 \%)$ accompanied with bone metastasis. It was indicated that myelosuppression was more significant in post-chemotherapy breast cancer patients with bone metastasis than patients without accompanied bone metastasis. ${ }^{37}$ Other toxic adverse reactions, mostly grade 1-2, cover rash, stomatitis, hepatic dysfunction, nausea and vomiting and others.

According to previous literature, the efficacy and prognosis of Non-TNBC are better than that of TNBC. Similar conclusions can be drawn from our study. The literature shows that the platinum-containing regimen is superior to the non-platinum-containing regimen in the treatment of TNBC. $\mathrm{Hu} \mathrm{XC}$ et al found that cisplatin combined with gemcitabine was superior to paclitaxel plus gemcitabine in a Phase III clinical trial of first-line treatment for advanced TNBC. The median progression-free survival (PFS) was 7.73 months vs 6.47 months, respectively, with a significant difference $(P<0.05){ }^{38}$ Zhang WX and other studies found that gemcitabine combined with cisplatin regimen achieved higher DCR in the treatment of advanced breast cancer in the second or third line. The DCR of the TNBC group was higher than that of the NonTNBC group, and the difference was statistically significant $(P<0.05) .{ }^{39}$ Hong RX and other studies have shown that platinum-containing regimens can prolong PFS and OS in patients with TNBC with primary lung metastases compared with platinum-free chemotherapy. All of the above studies suggest that platinum-containing regimens have a good effect on TNBC in the treatment of advanced breast cancer. ${ }^{40}$ Hierarchical analysis showed that TNBC was worse than Non-TNBC in terms of efficacy and prognosis. In addition, TTP and OS between groups with different line treatments showed statistically significant differences, consistent with other clinical trials and previous study reports. ${ }^{41-43}$

The scale of our study was small while the median PFS time was 5.0 months. Due to the longtime span of case collection, most patients received following treatments after progression of disease. By the end of follow-up, there were 43 deaths; the estimated median survival time was 28.5 months. The preliminary results of reference reports and this study have shown that the two-drug combination regimen containing lobaplatin shows a relatively high effective rate and DCR, in patients with metastatic or recurrent breast cancer, while the drug has a less toxic side effect. It is still effective for patients who have been treated with multiple chemotherapy or who are resistant to multiple drugs. Further clinical studies are required to offer MBC patients more benefits. 


\section{Conclusion}

Lobaplatin-based regimen chemotherapy for advanced MBC patients is effective and well tolerated.

\section{Ethical approval}

All procedures performed in studies involving human participants were in accordance with the ethical standards of the institutional and/or national research committee and with the 1964 Helsinki declaration and its later amendments or comparable ethical standards. Our study was approved by Ethics Committee of Jiangsu Cancer Hospital.

\section{Informed consent}

Verbal informed consent was obtained from all individual participants included in this study; for this type of study, written informed consent is not required.

\section{Disclosure}

The authors report no conflicts of interest in this work.

\section{References}

1. Heinemann V. Gemcitabine plus cisplatin for treatment of meta-static breast cancer. Clin Breast Cancer. 2002;3:24-29.

2. Decatris MP, Sundar S, O'Byrne KJ. Platinum-based chemotherapy in metastatic breast cancer: current status. Cancer Treat Rev. 2004;30:53-81. doi:10.1016/S0305-7372(03)00139-7

3. Sparano JA, Neuberg C, Glick JH, et al. Phase II trial of biweekly paclitaxel and cisplatin in advanced breast carcinoma: an Eastern Cooperative Oncology Group study. $J$ Clin Oncol. 1997;15:1880-1884. doi:10.1200/JCO.1997.15.5.1880

4. Gelmon KA, O'Reilly SE, Tolcher AW, et al. Phase I/II trial of biweekly paclitaxel and cisplatin in the treatment of metastatic breast cancer. $J$ Clin Oncol. 1996;14:1185-1191. doi:10.1200/ JCO.1996.14.4.1185

5. Mustacchi G, Muggia M, Milani S, et al. A phase II study of cisplatin and vinorelbine in patients with metastatic breast cancer. Ann Oncol. 2002;13:1730-1736. doi:10.1093/annonc/mdf290

6. Egger SJ, Willson ML, Morgan J, et al. Platinum-containing regimens for metastatic breast cancer. Cochrane Database Syst Rev. 2017;6:CD003374.

7. Rossi L, Biagioni C, McCartney A, et al. Platinum-based agent and fluorouracil in metastatic breast cancer: a retrospective monocentric study with a review of the literature. Anticancer Res. 2018;38 (8):4839-4845. doi:10.21873/anticanres.12795

8. Harstrick A, Bokemeyer C, Scharnofkse M, et al. Preclinical activity of a new platinum analogue, lobaplatin, in cisplatin-sensitive and resistant human testicular, ovarian, and gastric carcinoma cell lines. Cancer Chemother Pharmacol. 1993;33:43-47.

9. Gietema JA, Guchelaar HJ, de Vries EG, Aulenbacher P, Sleijfer DT, Mulder NH. A phase I study of lobaplatin (D-19466) administered by $72 \mathrm{~h}$ continuous infusion. Anticancer Drugs. 1993;4:51-55.

10. Wheate NJ, Walker S, Craig GE, Oun R. The status of platinum anticancer drugs in the clinic and in clinical trials. Dalton Trans. 2010;39:8113-8127. doi:10.1039/c0dt00292e

11. Jiang WQ, Lin TY, Xu RH, et al. Phase II clinical study of lobaplatin in the treatment of breast cancer. J Pract Cancer. 1998;13 (4):294-295.
12. Xu BH, Wei Y, Feng JF, et al. Clinical efficacy of lobaplatin combined with vinorelbine in the treatment of advanced breast cancer in 33 cases. J Clin Oncol. 2006;11(12):887-894.

13. Siegel RL, Miller KD, Jemal A. Cancer statistics, 2016. CA Cancer $J$ Clin. 2016;66(1):7-30. doi:10.3322/caac.21332

14. Early Breast Cancer Trialists' Collaborative Group (EBCTCG). Effects of chemotherapy and hormonal therapy for early breast cancer on recurrence and 15-year survival: an overview of the randomised trials. Lancet. 2005;365(9472):1687-1717. doi:10.1016/S01406736(05)66544-0

15. DeSantis C, Jemal A, Ward E, et al. Temporal trends in breast cancer mortality by state and race. Cancer Causes Control. 2008; $19: 537-545$.

16. AE tern a Laboratories. Lobaplatin (D-19466). Drugs R D. 2003;4 (6):369-372. doi:10.2165/00126839-200304060-00008

17. Harstric A, Hapke G, Scharno FM, et al. High activity of lobaplatin in cisplatin sensiutive and resistant human carcinoma cell lines in vitro and in vivo. Proc Sm Assoc Cancer Res. 1993;34:403.

18. Engel JB, Mavens T, Hahne JC, et al. Effects of lobaplatin as a single agent and in combination with TRAIL on the growth of triple negative p53 mutated breast cancers in vitro. Anticancer Drugs. 2012;23 (4):426-436. doi:10.1097/CAD.0b013e32834fb8ce

19. Mross K, Meyberg F, Fieberg HH, et al. Pharmacokinetic and pharmacodynamic study with lobaplatin (D-19466), a new platinum complex, after bolus administration. Onkologie. 1992;15:139-146.

20. McKeage MJ. Lobaplatin: a new antitumour platinum drug. Expert Opin Investig Drugs. 2001;10(1):119-128. doi:10.1517/ 13543784.10.1.119

21. Xie CY, Xu YP, Jin W, et al. Antitumor activity of lobaplatin alone or in combination with antitubulin agents in non small cell lung cancer. Anticancer Drugs. 2012;23(7):698-705. doi:10.1097/ CAD.0b013e328352cc10

22. Gietema JA, Veldhuis HJ, Guchelaar HJ, et al. Phase II and pharmacokinetic study of lobaplatin in patients with relapsed ovarian cancer. Br J Cancer. 1995;71:1302-1307. doi:10.1038/bjc.1995.252

23. Kavanagh JJ, Creightoon L, Edwards CL, et al. A trial of lobaplatin (D-19466) in platinum ovarian cancer. Gynecol Oncol. 1995;58:106-109.

24. Sternburg CN, De Mulder P, Fossa S, et al. Lobaplatin in advanced urothelial tract tumors. Ann Oncol. 1997;8:695-696. doi:10.1023/ a: 1008269432176

25. Jung P, Bachmann P, Burk K, Jakse G. Lobaplatin in combination with methotrexate and vinblastine in patients with transitional cell carcinoma of the urinary tract - a pilot phase I / II study. Eur J Cancer. 1995;31 a (11):1891-1892. doi:10.1016/0959-8049(95)00369-T

26. Lu D, Xu L, Shanshan C, et al. Lobaplatin for treatment of chronic myeloid leukemia (CML) - a pilot study. Blood. 1997;90(10S):276b, 3987.

27. Degardin M, Armand JP, Chevallier B, et al. A clinical screening cooperative group phase II evaluation of lobaplatin ASTA D-19466) in advanced head and neck cancer. Invest New Drugs. 1995; 13:253-255.

28. Welink J, Boven E, Ver Morken JB, et al. Pharmacokinetics and pharmacodynamics of lobaplatin (D-19466) in patients with advanced solid tumors, including patients with impaired renal of liver function. Clin Cancer Res. 1999;5:2349-2358.

29. Xu B, Yuan F, Feng J, et al. Clinical efficacy of lobaplatin combined with vinorelbine in the treatment of 33 patients with advanced breast cancer. J Clin Oncol. 2006;11(12):887-894.

30. Deng QQ, Huang XE, Li HY, et al. Phase II trial of Loubo® (Lobaplatin) and pemetrexed for patients with metastatic breast cancer not responding to anthracycline or taxanes. Asian Pac J Cancer Prev. 2013;14:413-417.

31. Wang Z, Xu L, Wang H, et al. Lobaplatin-based regimens outperform cisplatin for metastatic breast cancer after anthracyclines and taxanes treatment. Saudi J Biol Sci. 2018;25(5):909-916. doi:10.1016/j. sjbs.2018.01.011 
32. Wu X, Tang P, Li S, et al. A randomized and open-label phase II trial reports the efficacy of neoadjuvant lobaplatin inbreast cancer. Nat Commun. 2018;9(1):832. doi:10.1038/s41467-018-03210-2

33. Peng Y, Ye LIU, Xc REN, et al. A phase I clinical trial of dose escalation of lobaplatin in combination with fixed-dose docetaxel for the treatment of human solid tumours that had progressed following chemotherapy. Oncol Lett. 2015;9:67-74. doi:10.3892/ ol.2014.2675

34. Song L, He J, Zhang X, et al. Clinical observation of pemetrexed combined with lobaplatin in the treatment of advanced breast cancer with failure of anthracycline and taxane chemotherapy. J Clin Oncol. 2012;17(3):237-239.

35. Ma W, Zhang Q, Zhao W, et al. Clinical observation of lobaplatin combined with vinorelbine in the treatment of 32 cases of advanced breast cancer. J Clin Oncol. 2009;14(9):816-817.

36. Hao J, Ma Q, Liu P. A comparative study of gemcitabine combined with lobaplatin or cisplatin in the treatment of anthracycline and taxane-resistant advanced breast cancer. Clin Pharmacol Ther. 2012;17 (5):554-558.

37. Li Y, Zhang J, Shen L, et al. Clinical analysis of bone marrow suppression of breast cancer with bone metastases after chemotherapy. J Pract Oncol. 2013;28(3):287-291.
38. Hu XC, Zhang J, Xu BH, et al. Cisplatin plus gemcitabine versus paclitaxel plus gemcitabine as first-line therapy for metastatic triple-negative breast cancer (CBCSG006): a randomised, open-label, multicentre, phase 3 trial. Lancet Oncol. 2015;16 (4):436-446. doi:10.1016/S1470-2045(15)70064-1

39. Zhang WX, Zhang YZ, Zou FQ. Control study of gemcitabine combined with cisplatin in Sanyin and non-triple negative breast cancer. Chin J Cancer Prev Treat. 2013;20(22):1751-1754.

40. Hong RX, Ma F, Shi XQ, et al. Comparison of the efficacy of platinum-containing and platinum-free regimens in the treatment of first-negative lung metastases in triple-negative breast cancer. Chin J Oncol. 2014;36(10):788-792.

41. Gass P, Lux MP, Rauh C, et al. Prediction of pathological complete response and prognosis in patients with neoadjuvant treatment for triple-negative breast cancer. BMC Cancer. 2018;18(1):1051. doi:10.1186/s12885-018-4242-8

42. Walsh EM, Shalaby A, O'Loughlin M, et al. Outcome for triple negative breast cancer in a retrospective cohort with an emphasis on response to platinum-based neoadjuvant therapy. 2019;174(1);1-13.

43. Hm LI, Mx ZHANG, Lm NIU, et al. Efficacy and safety of lobaplatin combined chemotherapy in advanced breast cancer. Chin J Cancer Prev Treat. 2018;11:803-806.
OncoTargets and Therapy

\section{Publish your work in this journal}

OncoTargets and Therapy is an international, peer-reviewed, open access journal focusing on the pathological basis of all cancers, potential targets for therapy and treatment protocols employed to improve the management of cancer patients. The journal also focuses on the impact of management programs and new therapeutic

\section{Dovepress}

agents and protocols on patient perspectives such as quality of life, adherence and satisfaction. The manuscript management system is completely online and includes a very quick and fair peer-review system, which is all easy to use. Visit http://www.dovepress.com/ testimonials.php to read real quotes from published authors. 